\title{
Universal local operator quenches and entanglement entropy
}

\author{
Arpan Bhattacharyya, ${ }^{a, b}$ Tadashi Takayanagi $^{a, c}$ and Koji Umemoto ${ }^{a}$ \\ ${ }^{a}$ Center for Gravitational Physics, Yukawa Institute for Theoretical Physics, \\ Kyoto University, \\ Kyoto 606-8502, Japan \\ ${ }^{b}$ Indian Institute of Technology, \\ Gandhinagar, Gujarat 382355, India \\ ${ }^{c}$ Kavli Institute for the Physics and Mathematics of the Universe, \\ University of Tokyo, \\ Kashiwa, Chiba 277-8582, Japan \\ E-mail: abhattacharyya@iitgn.ac.in, takayana@yukawa.kyoto-u.ac.jp, \\ koji.umemoto@yukawa.kyoto-u.ac.jp
}

ABSTRACT: We present a new class of local quenches described by mixed states, parameterized universally by two parameters. We compute the evolutions of entanglement entropy for both a holographic and Dirac fermion CFT in two dimensions. This turns out to be equivalent to calculations of two point functions on a torus. We find that in holographic CFTs, the results coincide with the known results of pure state local operator quenches. On the other hand, we obtain new behaviors in the Dirac fermion CFT, which are missing in the pure state counterpart. By combining our results with the inequalities known for von-Neumann entropy, we obtain an upper bound of the pure state local operator quenches in the Dirac fermion CFT. We also explore predictions about the behaviors of entanglement entropy for more general mixed states.

KeYwords: AdS-CFT Correspondence, Conformal Field Theory, Black Holes

ArXiv EPRINT: 1909.04680 


\section{Contents}

1 Introduction 1

2 Review of pure state local operator quenches 3

3 Universal local operator quenches 5

3.1 Path-integral description 5

$\begin{array}{lll}3.2 & \text { Calculating entanglement entropy under universal local operator quench } & 7\end{array}$

4 Evolution of entanglement entropy in holographic CFTs 9

$\begin{array}{ll}4.1 & \text { High temperature phase }\end{array}$

$\begin{array}{lll}4.2 & \text { Low temperature phase } & 10\end{array}$

5 Evolution of entanglement entropy in free Dirac fermion CFT 10

$\begin{array}{lll}5.1 & \text { Case } 1: \beta=O(1) & 11\end{array}$

5.2 Case $2: \beta \ll 1 \quad 12$

6 Bounds for entanglement entropy 12

$\begin{array}{lll}\text { 6.1 Entanglement entropy bound for holographic CFTs } & 14\end{array}$

6.2 Entanglement entropy bound for Dirac fermion CFTs 14

7 Bounds for Rényi entropy and simple examples $\quad 14$

$\begin{array}{ll}7.1 \text { The second Rényi entropy } & 15\end{array}$

$\begin{array}{ll}7.2 & \text { The } n \text {-th Rényi entropy } \\ \end{array}$

8 More general mixed states in holographic CFTs $\quad 17$

$\begin{array}{llr}8.1 \text { Setup } & 18\end{array}$

$\begin{array}{lll}8.2 & \text { Case } 1: \gamma=\mathcal{O}(1) & 18\end{array}$

$\begin{array}{ll}8.3 \text { Case } 2: \gamma=\lambda c^{1-q} & 19\end{array}$

9 Conclusions 20

\section{Introduction}

Entanglement entropy characterizes the degrees of freedom in quantum field theories (QFTs) and thus plays a role of the universal order parameter in QFTs [1-3]. At the same time, entanglement entropy is an important probe of spacetime geometry via the AdS/CFT [4-6]. Properties of entanglement entropy in quantum field theories have been extensively studied so far. However, in most of the works so far, each of total systems is given by a pure state given by a vacuum or a certain excited state. Examples for which total systems are given by mixed states have been mostly limited to finite temperature states. The main purpose of this paper is to introduce an interesting new class of mixed states in conformal field theories (CFTs) and present calculations of entanglement entropy 
for such mixed states. Consider the von-Neumann entropy of a mixed state $\rho$, which is written as a linear combination:

$$
\rho=\sum_{i} p_{i} \rho_{i}
$$

where $\rho_{i}(i=1, \cdots, N)$ are density matrices and the coefficients $0<p_{i} \leq 1$ satisfy $\sum_{i} p_{i}=1$. The concavity inequality and another well-known inequality give the lower and upper bound of the entropy $S(\rho)$ as follows (refer to e.g. [7])

$$
\sum_{i} p_{i} S\left(\rho_{i}\right) \leq S(\rho) \leq \sum_{i} p_{i} S\left(\rho_{i}\right)+H(p)
$$

where $H(p)=-\sum_{i} p_{i} \log p_{i}$ is the Shannon entropy. The left inequality is saturated when $\rho_{i}$ are all identical, while the right one is saturated when $\rho_{i}$ are orthogonal to each other. The inequality (1.2) gives an important clue to understand properties of entanglement entropy for mixed states. To see this, consider a holographic setup where two classical gravity backgrounds $M_{1}$ and $M_{2}$ are dual to two pure quantum states $\rho^{(1)}=\left|\psi_{1}\right\rangle\left\langle\psi_{1}\right|$ and $\rho^{(2)}=\left|\psi_{2}\right\rangle\left\langle\psi_{2}\right|$, respectively. Let us ask what we can say about the mixed state $p \rho^{(1)}+$ $(1-p) \rho^{(2)}$. To study this, let us consider a reduced density matrix $\rho_{A}=p \rho_{A}^{(1)}+(1-p) \rho_{A}^{(2)}$ for a subsystem $A$. Since the Shannon entropy $H(p)$ is $O(1)$ and $S\left(\rho_{i}\right)$ is the order of the central charge $c$ (or equally the square of the rank of gauge group $N^{2}$ ), the inequality (1.2) approximately becomes an equality at the leading order of $1 / c=1 / N^{2}$ expansion:

$$
S\left(\rho_{A}\right) \simeq p S\left(\rho_{A}^{(1)}\right)+(1-p) S\left(\rho_{A}^{(2)}\right) .
$$

The same is true for a linear combination of states as long as the number of states is much smaller than $c$. This property in holographic CFTs (1.3) can be interpreted as the linearity of area operator in the AdS/CFT, which has been studied for explicit examples in [8].

However, when we take a linear combination of many states whose total numbers are $O(c)$, we cannot neglect the Shannon term and the inequality does not become tight. This is the case where we are interested in this paper.

In particular, we will choose $\rho_{i}$ to be a locally excited state in a CFT, created by acting a local operator $O_{i}(x)$ on a vacuum. This requires an appropriate regularization, which introduces an infinitesimally small parameter $s$. One interesting class of examples is provided by choosing $p_{i}$ to be Boltzmann-like distribution $p_{i} \propto e^{-\beta \Delta_{i}}$, where $\Delta_{i}$ is the conformal dimension of the operator $O_{i}(x)$. Note that this is different from the genuine Boltzmann distribution which describes a finite temperature state because $\Delta_{i}$ is not the energy of the locally excited state on $\mathrm{R}^{2}$ (i.e. a CFT on an infinite line). This defines a very simple but new class of (time-dependent) mixed states in CFTs in any dimensions.

In this paper we will focus on two dimensional CFTs on a plane $\mathrm{R}^{2}$ and analyze the properties of this class of mixed states, especially through computations of the entanglement entropy of $\rho_{A}=\sum p_{i} \rho_{A}^{(i)}$. This class of mixed states only depend on two parameters: $\beta$ and the regularization parameter $s$ of the local operator. Note that implicitly the result depends on the standard UV cut off (or lattice constant) denoted by $\epsilon$ as usual in the calculations of entanglement entropy. 
Even though the properties of each of locally excited states $\rho_{i}$ depend on the details of $O_{i}(x)$, the mixed states we consider only depend on the two parameters $\beta$ and $s$ as we mentioned. Motivated by this, we call this class of mixed states universal local operator quenches. We expect that properties of universal local quench states, e.g. the time evolutions of entanglement entropy, may provide a classification of CFTs, which can distinguish chaotic properties between different CFTs. This will be confirmed in the results of this paper.

This paper is organized as follows: In section 2, we present a brief review of local operator quenches based on each pure state. In section 3, we introduce the universal local operator quenches based on mixed states and describe the path-integral description as well as computations of entanglement entropy. In section 4, we study the time evolution of entanglement entropy under the universal local quenches in $2 \mathrm{~d}$ holographic CFTs. In section 5, we study the time evolution of entanglement entropy under the universal local quenches in the 2d Dirac Fermion CFT. In section 6, we analyze the consequence of the bounds (1.2) for our results of universal local quenches. In section 7, we discuss similar bounds for Rényi entropy and presents examples of explicit calculations of Rényi entropy for simple mixed states with local excitations. In section 8 , we explore the entanglement entropy for more general mixed states based on the inequality (1.2). In section 9 we summarize conclusions and discuss future problems.

\section{Review of pure state local operator quenches}

An instantaneous excitation at a point is called a local quench. A fundamental and important class of local quenches is the local operator quenches $[9,10]$, which is created by acting a local operator $O_{i}(x)$ on the vacuum (this pure state is denoted by $\rho_{i}$ ):

$$
\left|\Psi_{i}(t)\right\rangle=\mathcal{N}_{i} e^{-i t H} e^{-s H} O_{i}(x)|0\rangle,
$$

where $H$ is the CFT Hamiltonian. ${ }^{1}$ We write the conformal dimension of the operator $O_{i}(x)$ as $\Delta_{i}\left(=h_{i}+\bar{h}_{i}\right)$, where $h_{i}$ and $\bar{h}_{i}$ are its chiral and anti-chiral conformal dimension. We chose $\left\{O_{i}(x)\right\}$ to be an orthonormal basis such that

$$
\left\langle O_{i}(x) O_{j}(y)\right\rangle=\delta_{i j}\left(\frac{\epsilon}{x-y}\right)^{2 \Delta_{i}}
$$

where $\epsilon$ is the standard UV cut off (or lattice constant) denoted by $\epsilon$. The parameter $s$ in (2.1) is infinitesimally small and provides the regularization for the local quench procedure. ${ }^{2}$ The normalization factor $\mathcal{N}_{i}$ is chosen such that $\left\langle\Psi_{i}(t) \mid \Psi_{i}(t)\right\rangle=1$, which leads to $\mathcal{N}_{i}=\left(\frac{2 s}{\epsilon}\right)^{\Delta_{i}}$.

\footnotetext{
${ }^{1}$ There are other types of local quenches such as the joining local quenches [11] and the splitting local quenches [12]. The holographic dual of joining quenches is given by [13].

${ }^{2}$ This local quench regularization parameter $s$ should be distinguished from the ordinary UV cut off $\epsilon$, which is the lattice spacing. For example, the ground state entanglement entropy when the subsystem $A$ is an interval with the length $L$ is given by the well-known result $S_{A}^{(0)}=\frac{c}{3} \log \frac{L}{\epsilon}$.
} 
Here we are interested in the entanglement entropy for the locally excited state (2.1) for the subsystem $A$ defined by an interval $[a, b]$. The complement of $A$ is written as $B$. The entanglement entropy depends on the time $t$ as well as the regularization parameters $s$ and $\epsilon$. The entanglement entropy is computed as

$$
S_{A}=S\left(\rho_{A}^{(i)}\right),
$$

where $\rho_{i}$ is defined as

$$
\rho_{A}^{(i)}=\operatorname{Tr}_{B}\left[\left|\Psi_{i}(t)\right\rangle\left\langle\Psi_{i}(t)\right|\right]
$$

We can calculate the entanglement entropy $S\left(\rho_{A}^{(i)}\right)$ via the standard replica method:

$$
\begin{aligned}
S\left(\rho_{A}^{(i)}\right) & =-\left.\frac{\partial}{\partial n} \log \left[\operatorname{Tr}_{A}\left(\rho_{A}^{(i)}\right)^{n}\right]\right|_{n=1} \\
& =-\left.\frac{\partial}{\partial n} \log \left[\frac{\left\langle O_{i}(i s+t) O_{i}(-i s+t) \sigma_{n}(a) \bar{\sigma}_{n}(b)\right\rangle}{\left\langle O_{i}(i s+t) O_{i}(-i s+t)\right\rangle}\right]\right|_{n=1},
\end{aligned}
$$

where $\sigma_{n}$ denotes the twist operator. In particular, we take the large subsystem size limit and focus on the following time region:

$$
0<s \ll a \ll t \ll b .
$$

In the time region $a<t<b$, the entanglement entropy gets non-trivial because the excitations created by the local operator propagates into both the subsystem $A$ and $B$ at the same time, due to the left and right-moving modes at the speed of light.

In the papers $[9,10,14]$, this entropy was computed for the primary states for free CFTs and rational CFTs and it was found that the entropy is increased only by a finite amount, which coincides with the quantum dimension in 2d CFTs.

For holographic 2d CFTs, the entropy was computed in [15] using AdS/CFT, which was reproduced in [16] from the analysis for large $c$ CFT. Assuming that $\Delta_{i}$ is $O(c)$, this result in holographic CFTs reads

$$
S\left(\rho_{A}^{(i)}\right) \simeq \frac{c}{3} \log \frac{b-a}{\epsilon}+\frac{c}{6} \log \frac{t}{s}+\frac{c}{6} \log \left[\frac{1}{\sqrt{\frac{12 \Delta_{i}}{c}-1}} \sinh \left(\pi \sqrt{\frac{12 \Delta_{i}}{c}-1}\right)\right] .
$$

If $\Delta_{i}$ is much smaller than the central charge $c$, then the above formula gets modified. In particular if we take the limit $\Delta_{i} \rightarrow 0, S\left(\rho_{A}^{(i)}\right)$ coincides with the entanglement entropy for the ground state $[17]$

$$
S_{A}^{(0)}=\frac{c}{3} \log \frac{b-a}{\epsilon} .
$$

Note that the above result (2.7) in holographic CFTs is universal. The entanglement entropy only depends on the conformal dimension and is independent of the other details of the local operators. This behavior is peculiar to holographic CFTs, while the entanglement entropy in general CFTs depends on various details of local operators. ${ }^{3}$

\footnotetext{
${ }^{3}$ For a partial list of progress on properties of entanglement entropy under local operator quenches refer to $[12,18-42]$.
} 


\section{Universal local operator quenches}

Consider the following mixed state $\rho(\beta, s)$ as a local operator quench state:

$$
\rho(\beta, s)=\sum_{i} \frac{e^{-\beta\left(\Delta_{i}-\frac{c}{12}\right)}}{Z(\beta)} \cdot\left|\Psi_{i}(t)\right\rangle\left\langle\Psi_{i}(t)\right|,
$$

where

$$
Z(\beta)=\sum_{i} e^{-\beta\left(\Delta_{i}-\frac{c}{12}\right)}
$$

The indices $i$ run over all the quantum states in a given CFT including both primary and descendant states. The state $\left|\Psi_{i}(t)\right\rangle$ is given by (2.1) with the regularization parameter $s$.

Notice that this state is universally defined up to two parameters $\beta$ and $s$ for any CFTs in any dimensions. Therefore we call this a universal local operator quench. It is also useful to note that $Z(\beta)$ is the thermal partition function on a cylinder. Below we will study the behavior of entanglement entropy for this state in two dimensional CFTs.

\subsection{Path-integral description}

First we would like to provide a path-integral description of this mixed state $\rho(\beta, s)$ and the computations of the entanglement entropy $S_{A}=S\left(\rho_{A}\right)$, where $\rho_{A}=\operatorname{Tr}_{B}[\rho(\beta, s)]$. We introduce the complex coordinate $(X, \bar{X})$, where the universal local operator quench (3.1) will take place. We also write the coordinate as $X=x+i \tau$, where $\tau$ is the Euclidean time. We pick up an annulus region, called $\Sigma^{\prime}$ on this complex plane with two round disks (radius $l$ ) removed i.e. the region defined by

$$
\Sigma^{\prime}:\left|X-i r_{1}\right| \geq l, \quad\left|X+i r_{2}\right| \geq l
$$

where $r_{1}$ and $r_{2}$ are given by

$$
r_{1}=r-i t, \quad r_{2}=r+i t
$$

where $r$ is a parameter related to the regularization parameter of local quench as we will clarify later, and $t$ is the real time coordinate (we assume analytical continuation of the Euclidean time). These are summarized in the upper left picture in figure 1.

We find that the following conformal map from $X$ to $\zeta$ :

$$
\begin{aligned}
X & =i\left(\frac{\alpha_{1} \zeta+\alpha_{2}}{\zeta+1}\right), \\
\alpha_{1} & \equiv \frac{r_{1}-r_{2}}{2}-\sqrt{\frac{\left(r_{2}+r_{1}\right)^{2}}{4}-l^{2}}, \\
\alpha_{2} & \equiv \frac{r_{1}-r_{2}}{2}+\sqrt{\frac{\left(r_{2}+r_{1}\right)^{2}}{4}-l^{2}} .
\end{aligned}
$$


This transforms the two circles $\left|X-i r_{1}\right|=l$ and $\left|X+i r_{2}\right|=l$ into two concentric circles centered at the origin $|\zeta|=R_{2}$ and $|\zeta|=R_{1}$, where the two radii $R_{1}>R_{2}$ are

$$
\begin{aligned}
& R_{1}=\frac{l}{\frac{r_{1}+r_{2}}{2}-\sqrt{\frac{\left(r_{2}+r_{1}\right)^{2}}{4}-l^{2}}}, \\
& R_{2}=\frac{l}{\frac{r_{1}+r_{2}}{2}+\sqrt{\frac{\left(r_{2}+r_{1}\right)^{2}}{4}-l^{2}}} .
\end{aligned}
$$

Using (3.4), we can rewrite this as follows:

$$
\begin{array}{ll}
\alpha_{1}=-i t-\sqrt{r^{2}-l^{2}}, & \alpha_{2}=-i t+\sqrt{r^{2}-l^{2}}, \\
R_{1}=\frac{r+\sqrt{r^{2}-l^{2}}}{l}(>1), & R_{2}=\frac{r-\sqrt{r^{2}-l^{2}}}{l}(<1) .
\end{array}
$$

Notice also the relation $R_{1} R_{2}=1$.

Now we glue the two boundary circles $\left|X-i r_{1}\right|=l$ and $\left|X+i r_{2}\right|=l$ of the annulus $\Sigma^{\prime}$ (3.3) together and obtain a torus, denoted by $\Sigma$. A sketch of this torus $\Sigma$ in the coordinate $\zeta$ is depicted as the upper right picture in figure 1.

It is useful to further perform the standard transformation

$$
\zeta=e^{w}
$$

with $w=\rho+i \theta$. The annulus is now mapped into the torus whose coordinate takes values in

$$
\log R_{2} \leq \rho \leq \log R_{1}, \quad 0 \leq \theta \leq 2 \pi,
$$

as in the lower picture in figure 1.

We would like to argue that the universal local operator quench state $\rho(\beta, s)(3.1)$ is described by a path-integral on this torus $\Sigma$. Remember that the conformal transformations (3.5) and (3.9) map the region $\Sigma$ into a cylinder with the two end circles identified into a torus (refer to the upper pictures in figure 2). The length of the torus in the $\rho$ direction is

$$
\beta=\log \frac{R_{1}}{R_{2}}=\log \left[\frac{r+\sqrt{r^{2}-l^{2}}}{r-\sqrt{r^{2}-l^{2}}}\right],
$$

which is identified with $\beta$ in (3.1). In the torus picture, we would like to regard $\rho$ as the Euclidean time. It is useful to notice that the original time slice $\tau=0$ corresponds to $\rho=0$.

Our claim that the path-integral on this torus $\Sigma$ describes the universal local quench state $\rho(\beta, s)$ can be understood by comparing this setup with the path-integral description of pure state counterpart. We can regard the pure state local quench state $\left|\Psi_{i}(t)\right\rangle\left\langle\Psi_{i}(t)\right|(2.1)$ as the small hole limit $l \rightarrow 0$, with the identification $s=r$. As in lower pictures of figure 2, the pure state local quench is described by an infinitely long cylinder where the state $|i\rangle$, dual to the operator $O_{i}$ via the usual state/operator correspondence, propagates.

To see more details, it is important to keep track of the lattice spacing. We write the lattice spacing in the original coordinate $X$ as $\epsilon$. Note that $\epsilon$ takes the same value 


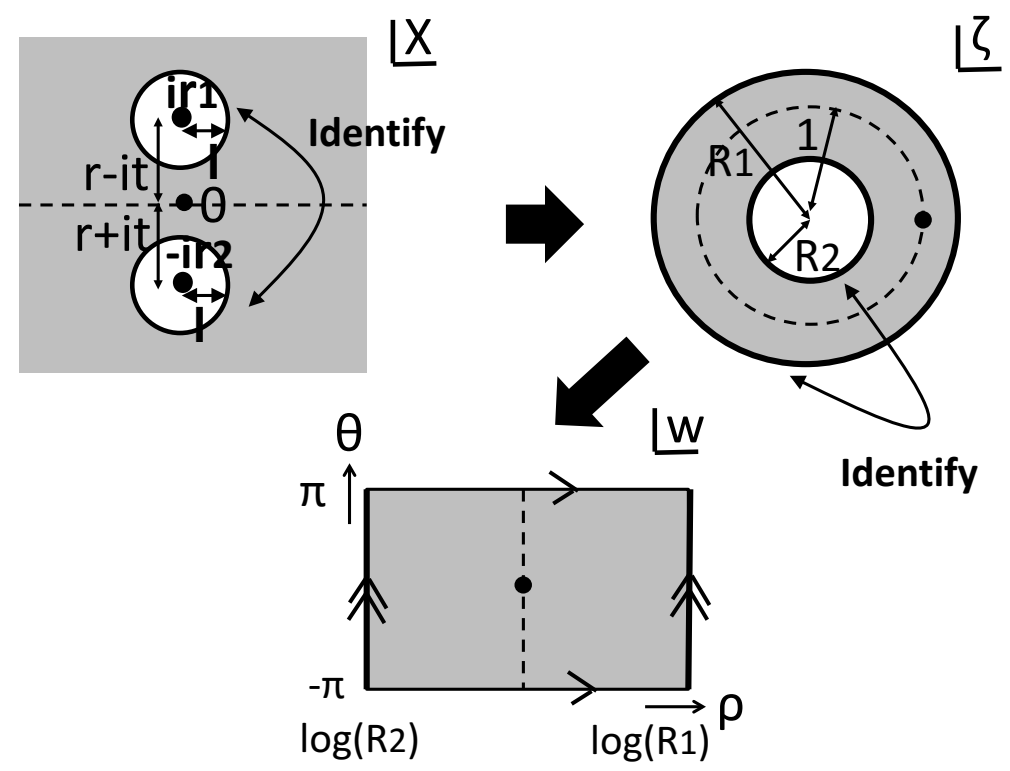

Figure 1. The path-integral on a torus $\Sigma$ which describes a mixed state local quench and its conformal transformations into $\zeta$ and $w$ coordinate from the original $X$ coordinate.

everywhere in this coordinate. This cut off is mapped into that in the torus coordinate $w$, which is denoted by $\delta$. As follows from our conformal transformations (3.5) and (3.9), we find that the cut off $\epsilon$ is related to $\delta$ :

$$
\delta=\frac{2 \sqrt{r^{2}-l^{2}}}{\sqrt{\left((x+t)^{2}+r^{2}-l^{2}\right)\left((x-t)^{2}+r^{2}-l^{2}\right)}} \cdot \epsilon .
$$

Similarly, the cut off relation for the pure state local quench reads

$$
\delta=\frac{2 s}{\sqrt{\left((x+t)^{2}+s^{2}\right)\left((x-t)^{2}+s^{2}\right)}} \cdot \epsilon
$$

Therefore, the cut off $\delta$ for the universal local operator quench state $\rho(\beta, s)$ (upper picture of figure 2) is related to that for the pure state one $\left|\Psi_{i}(t)\right\rangle\left\langle\Psi_{i}(t)\right|$ (lower picture of figure 2) via the identification of the quench parameter

$$
s=\sqrt{r^{2}-l^{2}} .
$$

In summary, after the conformal maps, the state for the path-integration over the torus $\Sigma$ can be identified with the state $\rho(\beta, s)$ defined by (3.1), where the parameters $\beta$ and $s$ are related to $r$ and $l$ in the path-integral description by (3.11) and (3.14), respectively.

\subsection{Calculating entanglement entropy under universal local operator quench}

Now we move back to the original problem of computing entanglement entropy for the universal local operator quench states. We choose the subsystem $A$ to be an interval $x \in[a, b]$ on the real axis $\tau=0$, which is mapped into an interval on the circle $\rho=0$. The UV cut off (3.12) in the torus coordinate which corresponds to the original one $\epsilon$ is denoted by $\delta_{a}$ and $\delta_{b}$ at the two end points of the interval $A$ i.e. $x=a$ and $x=b$, respectively. 

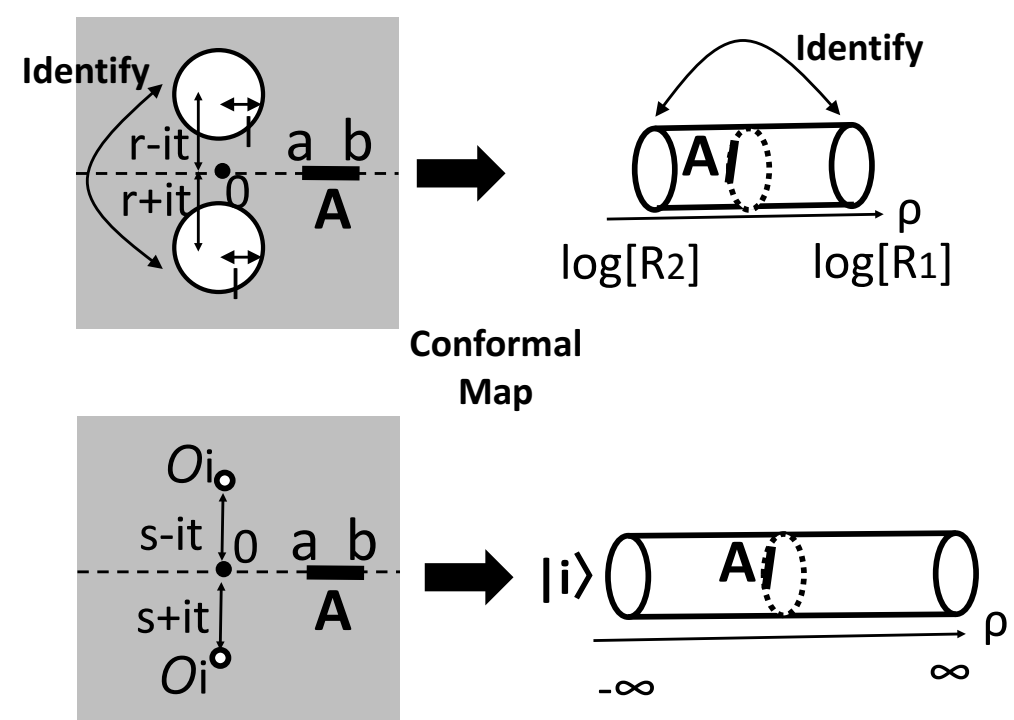

Figure 2. Comparison between the universal (mixed state) local quench (3.1), depicted in the upper picture and the pure state local quench (2.1), depicted in the lower picture. We also expressed the interval $A$ for which we calculate the entanglement entropy as the thick lines.

The entanglement entropy $S_{A}$ can be computed using the standard replica trick, i.e. by taking derivative w.r.t. $n$ of the trace:

$$
S_{A}=-\left.\frac{\partial}{\partial n} \log \left[\operatorname{Tr}_{A} \rho^{n}\right]\right|_{n=1}=-\left.\frac{\partial}{\partial n} \log \left\langle\sigma_{n}(a) \overline{\sigma_{n}}(b)\right\rangle_{\Sigma}\right|_{n=1}
$$

In this way, we can calculate the entanglement entropy from the two point function on a torus.

In particular, we are interested in the middle time region of the large subsystem limit (2.6). In this region we can simplify the cut offs $\delta_{a}$ and $\delta_{b}$ in the torus coordinate as follows:

$$
\delta_{a} \simeq \frac{2 \sqrt{r^{2}-l^{2}}}{t^{2}} \cdot \epsilon, \quad \delta_{b} \simeq \frac{2 \sqrt{r^{2}-l^{2}}}{b^{2}} \cdot \epsilon .
$$

The endpoints of the subsystem $A$ given by $(x, \tau)=(a, 0)$ and $(b, 0)$ are mapped into the points $(\rho, \theta)=\left(0, \theta_{a}\right)$ and $\left(0, \theta_{b}\right)$, where $\theta_{a, b}$ can be found from the conformation maps (3.5) and (3.9). Here we have to be careful about the analytical continuation of the Euclidean time $\tau$ to the real time by the identification $\tau=i t$. For this we write the 'imaginary part' of $w$ and $-\bar{w}$ as $\theta$ and $\bar{\theta}$, respectively. Note that in general we have $\theta \neq \bar{\theta}$ except at $t=0$. In the middle time region (2.6) with the large subsystem size limit, we find

$$
\begin{aligned}
& \theta_{a} \simeq-\pi+\frac{2 \sqrt{r^{2}-l^{2}}}{t}, \quad \bar{\theta}_{a} \simeq \pi-\frac{2 \sqrt{r^{2}-l^{2}}}{t}, \\
& \theta_{b} \simeq \bar{\theta}_{b} \simeq \pi-\frac{2 \sqrt{r^{2}-l^{2}}}{b} .
\end{aligned}
$$

Therefore we can rewrite the two point function of the twist operators on the original coordinate $(x, \tau)$ in terms of that on the torus coordinate $(\rho, \theta)$ by taking into account the 

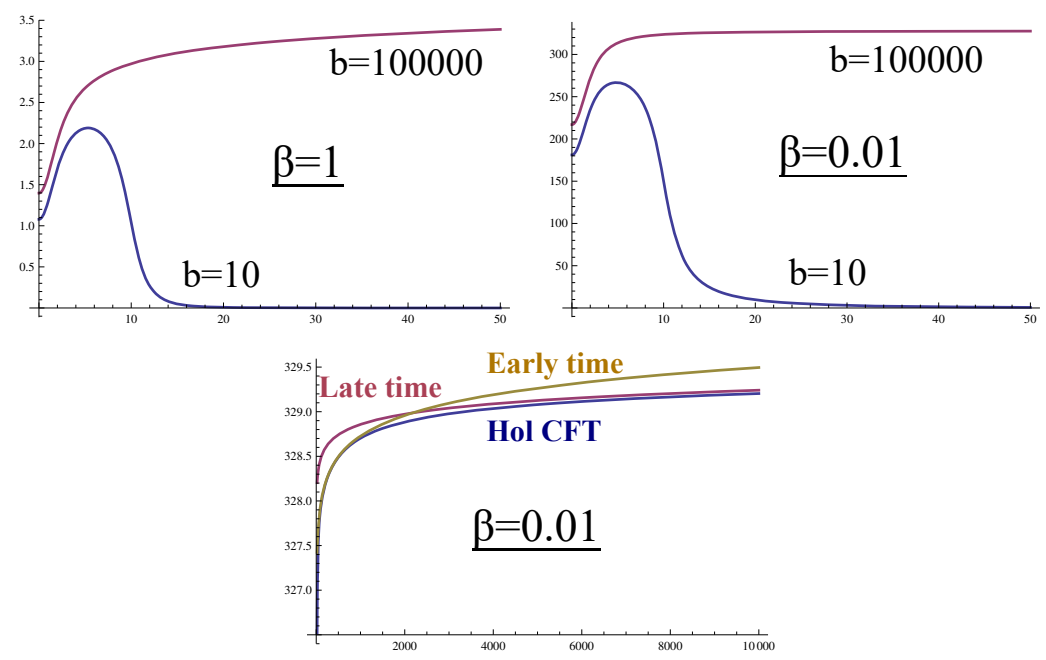

Figure 3. The plot of $\Delta S_{A}=S_{A}-S_{A}^{(0)}$ as a function of time $t$ in holographic CFTs. We set $c=1$ in all plots. The left and middle graph shows the plot for $\beta=1$ and $\beta=0.01$, respectively, both in the high temperature phase. We took $a=1, r=2$ and $l=1$. The blue and red graph correspond to $b=10$ and $b=100000$. The right picture shows the actual entanglement entropy (blue graph), the late time approximation (4.4) of the entanglement entropy (red graph), and the early time approximation (4.6) of the entanglement entropy (yellow graph), for $\beta=0.01, a=1$, $b=100000, r=2$ and $l=1$.

transformation (3.16) of the cut off scale. The further analysis of the two point functions on the torus depends on what kind of 2d CFTs we consider.

\section{Evolution of entanglement entropy in holographic CFTs}

In the holographic CFTs, there is a sharp phase transition for the partition function on a torus. We have the following two phases:

(i) High temperature phase : $\beta<2 \pi$ or, equally $\frac{l}{r}>\frac{1}{\cosh (\pi)}$

(ii) Low temperature phase : $\beta>2 \pi$ or, equally $\frac{l}{r}<\frac{1}{\cosh (\pi)}$.

The phase $(i)$ is dual to the BTZ black hole with the temperature $T=1 / \beta$. On the other hand, the phase $(i i)$ is dual to the thermal AdS geometry at the same temperature. In both backgrounds, the spatial coordinate $\theta$ is compactified as $\theta \sim \theta+2 \pi$.

\subsection{High temperature phase}

In the phase $(i)$ i.e. $\beta<2 \pi$, the entanglement entropy is computed by regarding the torus as an infinitely long cylinder extending into the $\theta$ direction with the coordinate $\rho$ compactified as $\rho \sim \rho+\beta$. Thus we find

$$
S_{A}=\frac{c}{6} \log \left[\frac{\beta^{2}}{\pi^{2} \delta_{a} \delta_{b}} \sinh \left(\frac{\pi\left(\theta_{a}-\theta_{b}\right)}{\beta}\right) \sinh \left(\frac{\pi\left(\bar{\theta}_{a}-\bar{\theta}_{b}\right)}{\beta}\right)\right] .
$$


Let us first assume $\beta$ is $O(1)$. For the time region (2.6), we can plug (3.17) into (4.2) and obtain the time evolution as follows:

$$
S_{A} \simeq \frac{c}{3} \log \left[\frac{\beta b t}{2 \pi \epsilon \sqrt{r^{2}-l^{2}}}\right]+\frac{c}{6} \log \sinh \left[\frac{2 \pi \sqrt{r^{2}-l^{2}}}{\beta t}\right]+\frac{c}{6} \log \sinh \left[\frac{2 \pi^{2}}{\beta}-\frac{2 \pi \sqrt{r^{2}-l^{2}}}{\beta t}\right] .
$$

Thus we obtain (we used the relation (3.14))

$$
S_{A} \simeq \frac{c}{3} \log \frac{b}{\epsilon}+\frac{c}{6} \log \frac{t}{s}+\frac{c}{6} \log \left[\frac{\beta}{2 \pi} \sinh \left(\frac{2 \pi^{2}}{\beta}\right)\right] .
$$

Refer to the left picture in figure 3 for a plot of $\Delta S_{A}$, namely the difference between the entanglement entropy for our excited state and that for the ground state (2.8).

If $\beta$ is very small $\beta \ll 1$ (i.e. high temperature limit), there is an additional intermediate behavior in the time region

$$
s \ll t \ll \frac{s}{\beta},
$$

where we find

$$
S_{A} \simeq \frac{c}{3} \log \frac{b}{\epsilon}+\frac{c}{3} \log \left(\frac{\beta t}{4 \pi s}\right)+\frac{\pi^{2} c}{3 \beta} .
$$

However, for $t \gg \frac{s}{\beta}$ we find the previous behavior (4.3). Notice that the coefficient of $\log t$ is doubled in (4.6) compared with the previous one (4.3). Refer to the middle and right picture in figure 3 for a plot.

\subsection{Low temperature phase}

In the phase $(i i)$ i.e. $\beta>2 \pi$, the entanglement entropy is computed by regarding the torus as an infinitely long cylinder extending in the $\rho$ direction with the coordinate $\theta$ compactified as $\theta \sim \theta+2 \pi$. Thus we obtain

$$
S_{A}=\frac{c}{6} \log \left[\frac{4}{\delta_{a} \delta_{b}} \sin \left(\frac{\theta_{a}-\theta_{b}}{2}\right) \sin \left(\frac{\bar{\theta}_{a}-\bar{\theta}_{b}}{2}\right)\right] .
$$

By explicit calculations, we find that the entanglement entropy remains the same as that for the ground state:

$$
S_{A}=\frac{c}{3} \log \frac{b-a}{\epsilon},
$$

at any time $t$. Therefore there is no non-trivial time evolution in the low temperature phase within the large $c$ approximation.

\section{Evolution of entanglement entropy in free Dirac fermion CFT}

As another example, which is extremely different from the holographic CFTs, we would like to calculate the entanglement entropy $S\left(\rho_{A}\right)$ in the massless Dirac fermion CFT $(c=1)$ in the same setup. The entanglement entropy on a torus in this CFT was computed in [43]. In our present computation, we need to further perform the analytical continuation of the 
time and convert the UV cut off $\delta_{a . b}$ on the torus into the cut off $\epsilon$ for the original $\Sigma$ space via (3.17).

The entanglement entropy in our setup is obtained from the result in [43] as follows:

$$
S_{A}=\frac{1}{6} \log \left[\frac{\beta^{2}}{\pi^{2} \delta_{a} \delta_{b}}\right]+g\left(\theta_{b}-\theta_{a}\right)+g\left(\bar{\theta}_{b}-\bar{\theta}_{a}\right),
$$

where the function $g(y)$ is defined by

$$
\begin{aligned}
g(y)= & \frac{1}{6} \log \sinh \left(\frac{\pi y}{\beta}\right)+\frac{1}{6} \sum_{m=1}^{\infty} \log \left[\frac{\left(1-e^{\frac{2 \pi y}{\beta}} e^{-\frac{4 \pi^{2} m}{\beta}}\right)\left(1-e^{-\frac{2 \pi y}{\beta}} e^{-\frac{4 \pi^{2} m}{\beta}}\right)}{\left(1-e^{-\frac{4 \pi^{2} m}{\beta}}\right)\left(1-e^{-\frac{4 \pi^{2} m}{\beta}}\right)}\right] \\
& +\sum_{p=1}^{\infty} \frac{(-1)^{p}}{p} \cdot \frac{\left(\frac{\pi p y}{\beta}\right) \operatorname{coth}\left(\frac{\pi p y}{\beta}\right)-1}{\sinh \left(\frac{\pi p y}{\beta}\right)}
\end{aligned}
$$

It is useful to examine the behaviors of $g(y)$. In the limit $y \rightarrow 0$ we have

$$
g(y) \simeq \frac{1}{6} \log \frac{\pi y}{\beta}+O(y) .
$$

On the other hand, in the limit $y \rightarrow 2 \pi$ we find

$$
g(y) \simeq \frac{1}{6} \log \left[\frac{\pi}{\beta}(2 \pi-y)\right]+\frac{\pi^{2}}{3 \beta}+\sum_{p=1}^{\infty} \frac{(-1)^{p}}{p} \frac{\left(\frac{2 \pi^{2} p}{\beta}\right) \operatorname{coth}\left(\frac{2 \pi^{2} p}{\beta}\right)-1}{\sinh \left(\frac{2 \pi^{2} p}{\beta}\right)}+O(2 \pi-y) .
$$

Below we consider the evolution of entanglement entropy in the middle time region with the large subsystem size limit (2.6).

\subsection{Case 1: $\beta=O(1)$}

First we assume $\beta=O(1)$. In this case for the time region (2.6), we find the behavior

$$
S_{A} \simeq \frac{1}{3} \log \frac{b}{\epsilon}+\frac{1}{2} S_{t h}(\beta),
$$

where $S_{t h}(\beta)$ is the thermal entropy of the free Dirac fermion CFT at temperature $T=1 / \beta$ :

$$
S_{t h}(\beta)=\frac{2 \pi^{2}}{3 \beta}+2 \sum_{p=1}^{\infty} \frac{(-1)^{p}}{p} \frac{\left(\frac{2 \pi^{2} p}{\beta}\right) \operatorname{coth}\left(\frac{2 \pi^{2} p}{\beta}\right)-1}{\sinh \left(\frac{2 \pi^{2} p}{\beta}\right)} .
$$

Therefore, after the time $t=a$, when the signal of local operator quench started to affect $S_{A}$, the entanglement entropy quickly increases by a half of the thermal entropy and stays the same. Refer to the left picture in figure 4 for a comparison with the holographic result. 

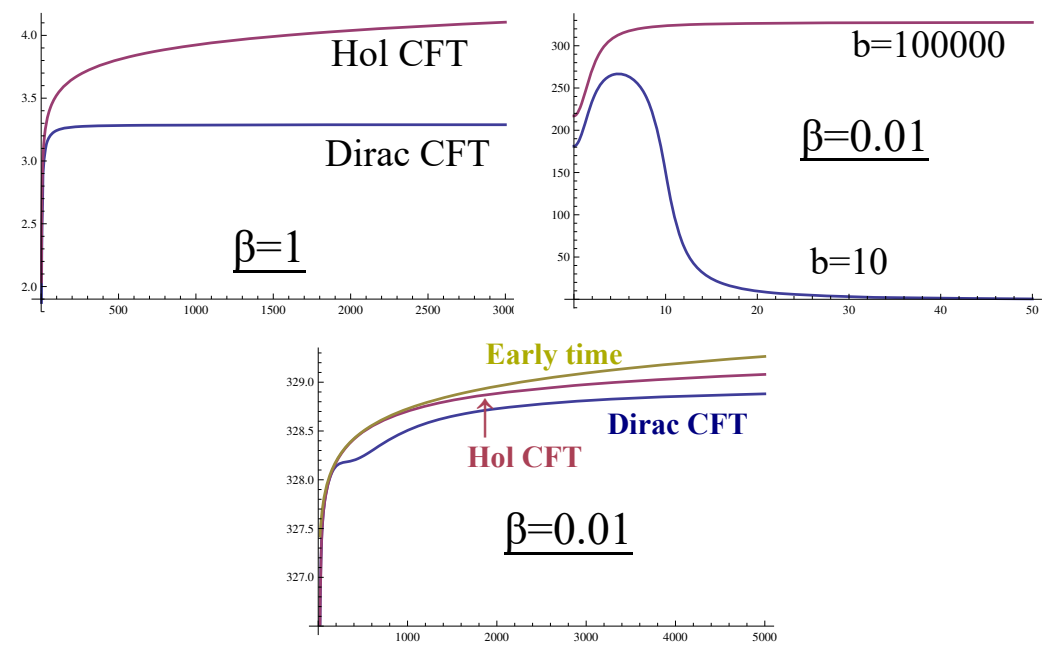

Figure 4. The plot of $\Delta S_{A}=S_{A}-S_{A}^{(0)}$ as a function of time $t$ for the Dirac fermion CFT. The left picture is the comparison between the Dirac fermion CFT result (blue graph) and the holographic CFT result (red graph) at $\beta=1, a=1, b=100000, r=2$ and $l=1$. The middle graph shows the plot in the Dirac fermion CFT for $\beta=0.01, a=1, r=2$ and $l=1$. The blue and red graph correspond to the two difference values of $b: b=10$ and $b=100000$, respectively. The right picture shows $\Delta S_{A}$ in the Dirac fermion CFT (Blue graph), the holographic CFT result (red graph), and the early time approximation (4.6) entanglement entropy (yellow graph) for $\beta=0.01$, $a=1, b=100000, r=2$ and $l=1$.

\subsection{Case $2: \beta \ll 1$}

On the other hand, if we assume the very high temperature region $\beta \ll 1$, there is a time region:

$$
s \ll t \ll \frac{s}{\beta},
$$

where we have $\frac{2 \pi}{\beta}(2 \pi-y) \gg 1$. Therefore, in this time period, we find the entanglement entropy behaves as

$$
S_{A} \simeq \frac{1}{3} \log \frac{b}{\epsilon}+\frac{1}{3} \log \left(\frac{\beta t}{4 \pi s}\right)+\frac{\pi^{2}}{3 \beta} .
$$

Note that this is identical to the holographic CFT result in the same time zone as in (4.6).

At the late time $t \gg \frac{s}{\beta}$, we find the previous behavior (5.5). Note also at high temperature $\beta \ll 1$ we have $S_{t h}(\beta) \simeq \frac{\pi^{2}}{3 \beta}$. Refer to the middle and right picture in figure 4 for explicit numerical plots.

\section{Bounds for entanglement entropy}

We would like to study the implications of the bounds (1.2) for entanglement entropy under the local operator quenches. We can rewrite the inequalities (1.2) as follows:

$$
S(\rho)-H(p) \leq \sum_{i} p_{i} S\left(\rho_{i}\right) \leq S(\rho)
$$


This inequality is useful when $S(\rho)$ is calculable e.g. the case where $\rho$ is a canonical distribution or our universal local quench. We can obtain the upper and lower bound for $S\left(\rho_{i}\right)$ i.e. the entropy for microscopic states or the pure state local operator quench states.

To apply the entropy bound to our example of operator local quenches, let us study the case where $p_{i}$ is given by the canonical distribution as in our universal local quenches:

$$
p_{i}=\frac{e^{-\beta\left(\Delta_{i}-\frac{c}{12}\right)}}{Z(\beta)}
$$

The Shannon entropy is given by the thermal entropy as $H(p)=S_{t h}(\beta)$. The microstates $\rho_{i}$ are now the reduced density matrices $\rho_{A}^{(i)}$ defined in (2.4). We identify the density matrix $\rho$ in (6.1) with the reduced matrix $\rho_{A}=\operatorname{Tr}_{B}[\rho(\beta, s)]$, which is described as the mixed state

$$
\rho_{A}=\sum_{i} \frac{e^{-\beta\left(\Delta_{i}-\frac{c}{12}\right)}}{Z(\beta)} \cdot \rho_{A}^{(i)} .
$$

This leads to the bounds from (6.1):

$$
S\left(\rho_{A}\right)-S_{t h}(\beta) \leq \sum_{i} \frac{e^{-\beta\left(\Delta_{i}-\frac{c}{12}\right)}}{Z(\beta)} S\left(\rho_{A}^{(i)}\right) \leq S\left(\rho_{A}\right) .
$$

If we write the density of states as $D(\Delta)$, the partition function looks like

$$
Z(\beta)=\sum_{\Delta} D(\Delta) e^{-\beta\left(\Delta-\frac{c}{12}\right)} .
$$

By imposing the modular invariance $Z(\beta)=Z\left(4 \pi^{2} / \beta\right)$, we find in the limit $\beta \rightarrow 0$,

$$
Z(\beta) \sim e^{\frac{\pi^{2} c}{3 \beta}}
$$

This leads to the Cardy formula as usual:

$$
D(\Delta) \sim e^{2 \pi \sqrt{\frac{c}{3}\left(\Delta-\frac{c}{12}\right)}} .
$$

For generic unitary CFTs, this Cardy formula (6.7) can be applied when $\Delta \gg c$. However, for holographic CFTs we can apply the Cardy formula (6.7) when $\Delta>\frac{c}{6}$ [44].

In the high temperature phase (i.e. when we can apply the Cardy formula), we can apply the saddle point approximation to an expectation value of a quantity $S(\Delta)$ :

$$
\frac{1}{Z(\beta)} \sum_{\Delta} D(\Delta) S(\Delta) e^{-\beta\left(\Delta-\frac{c}{12}\right)} \simeq S\left(\Delta_{\beta}\right)
$$

where we defined

$$
\Delta_{\beta}=\frac{c}{12}+\frac{\pi^{2} c}{3 \beta^{2}}
$$

or equally we have

$$
\beta \simeq \frac{2 \pi}{\sqrt{\frac{12 \Delta_{\beta}}{c}-1}} .
$$

Thus at high temperature, the bound (6.1) can be rewritten as follows:

$$
S_{A}^{U L O Q}(\beta)-S_{t h}(\beta) \leq S_{A}^{L O Q}\left(\Delta_{\beta}\right) \leq S_{A}^{U L O Q}(\beta) .
$$




\subsection{Entanglement entropy bound for holographic CFTs}

In the high temperature phase of holographic CFTs, by comparing (4.4) with (2.7) using (6.10), we find that the latter inequality of (6.4) is saturated i.e.

$$
S_{A}^{U L O Q}(\beta)=S_{A}^{L O Q}\left(\Delta_{\beta}\right) .
$$

This is consistent with the indistinguishability of the reduced density matrices $\rho_{A}^{(i)}$ as we trace out a larger part $B$. This is analogous to the result in [45] for the examples of a finite temperature mixed state versus each micro states.

On the other hand, in the low temperature phase, we have $S_{t h}(\beta)=O(1)$ due to the confining nature. Therefore, the inequalities (6.1) are saturated, though we cannot apply (6.12) as the saddle point approximation breaks down at low temperature. As we explained before, the entanglement entropy of the universal local operator quench state, becomes trivial in low temperature phase as in (4.8). This is consistent because in the discrete sum we find $e^{-\beta \Delta} \sim e^{-O(c)}$ is exponentially small and thus we find $\sum_{i} \frac{e^{-\beta\left(\Delta_{i}-\frac{c}{12}\right)}}{Z(\beta)} S\left(\rho_{A}^{(i)}\right)$ is approximated by the ground state entanglement entropy.

\subsection{Entanglement entropy bound for Dirac fermion CFTs}

In the Dirac fermion CFT, we find $\Delta S_{A}^{U L O Q}(\beta) \leq \frac{1}{2} S_{t h}(\beta)$ from the previous result (5.5). Therefore the bound (6.11) does not look tight. Nevertheless we obtain the upper bound

$$
\Delta S_{A}^{L O Q}(\Delta) \leq \frac{\pi^{2}}{3 \beta} \simeq \frac{\pi}{6} \sqrt{\frac{12 \Delta}{c}-1}
$$

In particular, this shows there should be no logarithmic time growth at late time in the Dirac fermion CFT as opposed to that in holographic CFTs.

\section{Bounds for Rényi entropy and simple examples}

The bounds for entanglement entropy (1.2) can be generalized to the Rényi entropy. First, we can generalize the lower bound (1.2) by replacing the von-Neumann entropy with the Rényi entropy for $\alpha \in(0,1]$,

$$
\sum_{i} p_{i} S_{\alpha}\left(\rho_{i}\right) \leq S_{\alpha}(\rho)(0<\alpha \leq 1)
$$

where $S_{\alpha}(\rho) \equiv 1 /(1-\alpha) \log \operatorname{Tr} \rho^{\alpha}$. However, it is well known that the Rényi entropy does not satisfy the concavity $\sum_{i} p_{i} S_{\alpha}\left(\rho_{i}\right) \leq S_{\alpha}(\rho)$ for $\alpha>1$. This bound is saturated if the microstates $\rho_{i}$ are indistinguishable (regardless to $\alpha$ ).

On the other hand, the upper bound by classical Rényi entropy $H_{\alpha}(p)=1 /(1-$ $\alpha) \log \left(\sum_{i} p_{i}^{\alpha}\right)$ is always true for any $\alpha$ when $\rho_{i}$ are pure (refer to e.g. [46]),

$$
S_{\alpha}(\rho) \leq \sum_{i} p_{i} S_{\alpha}\left(\rho_{i}\right)+H_{\alpha}(p)=H_{\alpha}(p) .
$$

In order to compute the Rényi entropy $S_{n}\left(\rho_{A}\right)$ for the universal local operator quench state (3.1) for arbitrary $n \in \mathbb{Z}$, one needs to deal with higher genus partition functions. 
Here instead we deal with a simpler example of mixed state which is given by the mixture of two locally excited states

$$
\begin{aligned}
\rho_{A}(t) & =\operatorname{Tr}_{B}\left[p\left|O_{1}(t)\right\rangle\left\langle O_{1}(t)|+(1-p)| O_{2}(t)\right\rangle\left\langle O_{2}(t)\right|\right] \\
& \equiv p \rho_{1}(t)+(1-p) \rho_{2}(t)
\end{aligned}
$$

where $0 \leq p \leq 1$. We will take $O_{1}=e^{i \eta \phi}$ and $O_{2}=e^{-i \eta \phi}$ with $h_{1}=\bar{h}_{1}=h_{2}=\bar{h}_{2}=\frac{\eta^{2}}{2}$ in a free scalar field theory. The local operators are inserted at $X_{1} \equiv X_{3} \equiv i r_{1}$ and $X_{2} \equiv X_{4} \equiv-i r_{2}$ in the $X$ plane (we have used $r=s$ for $l \rightarrow 0$ ).

\subsection{The second Rényi entropy}

The second Rényi entropy is given by

$$
S_{2}\left(\rho_{A}\right)=-\log \left[p^{2} \operatorname{Tr} \rho_{1}^{2}+2 p(1-p) \operatorname{Tr}\left(\rho_{1} \rho_{2}\right)+(1-p)^{2} \operatorname{Tr} \rho_{2}^{2}\right] .
$$

We can compute these traces by using the following conformal map from $X$-plane to $z$-plane (see [14]),

$$
z^{2}=\frac{X-a}{X-b}
$$

and for the interacting term, we get

$$
\begin{aligned}
\operatorname{Tr} \rho_{1} \rho_{2} & =\frac{\left\langle O_{1}^{\dagger}\left(X_{1}, \bar{X}_{1}\right) O_{1}\left(X_{2}, \bar{X}_{2}\right) O_{2}^{\dagger}\left(X_{3}, \bar{X}_{3}\right) O_{2}\left(X_{4}, \bar{X}_{4}\right)\right\rangle}{\left\langle O_{1}^{\dagger}\left(X_{1}, \bar{X}_{1}\right) O_{1}\left(X_{2}, \bar{X}_{2}\right)\right\rangle\left\langle O_{2}^{\dagger}\left(X_{3}, \bar{X}_{3}\right) O_{2}\left(X_{4}, \bar{X}_{4}\right)\right\rangle} \frac{Z_{(2)}}{\left(Z_{(1)}\right)^{2}} \\
& =\prod_{k=1}^{4}\left(\frac{d z_{k}}{d X_{k}}\right)^{h_{k}}\left(\frac{d \bar{z}_{k}}{d \bar{X}_{k}}\right)^{\bar{h}_{k}} \frac{\left\langle O_{1}^{\dagger}\left(z_{1}, \bar{z}_{1}\right) O_{1}\left(z_{2}, \bar{z}_{2}\right) O_{2}^{\dagger}\left(z_{3}, \bar{z}_{3}\right) O_{2}\left(z_{4}, \bar{z}_{4}\right)\right\rangle}{\left\langle O_{1}^{\dagger}\left(X_{1}, \bar{X}_{1}\right) O_{1}\left(X_{2}, \bar{X}_{2}\right)\right\rangle\left\langle O_{2}^{\dagger}\left(X_{3}, \bar{X}_{3}\right) O_{2}\left(X_{4}, \bar{X}_{4}\right)\right\rangle} \frac{Z_{(2)}}{\left(Z_{(1)}\right)^{2}}
\end{aligned}
$$

where

$$
\begin{aligned}
z_{1} & =-z_{3}=\sqrt{\frac{a-t-i s}{b-t-i s}}, & z_{2} & =-z_{4}=\sqrt{\frac{a-t+i s}{b-t+i s}} . \\
\bar{z}_{1} & =-\bar{z}_{3}=\sqrt{\frac{a+t+i s}{b+t+i s}}, & \bar{z}_{2} & =-\bar{z}_{4}=\sqrt{\frac{a+t-i s}{b+t-i s}} .
\end{aligned}
$$

For the operators $O_{1}=e^{i \eta \phi}$ and $O_{2}=e^{-i \eta \phi}$, we find

$$
\frac{\left(Z_{(1)}\right)^{2}}{Z_{(2)}} \operatorname{Tr} \rho_{1} \rho_{2}=(1-z)^{4 h}(1-\bar{z})^{4 h},
$$

where $z$ and $\bar{z}$ are the conformal cross-ratios

$$
z=\frac{z_{12} z_{34}}{z_{13} z_{24}}, \quad \bar{z}=\frac{\bar{z}_{12} \bar{z}_{34}}{\bar{z}_{13} \bar{z}_{24}}
$$

where $z_{i j}=z_{i}-z_{j}$. In the $s \rightarrow 0$ limit, we have two regimes depending on $t$ [14]

$$
(z, \bar{z}) \rightarrow\left\{\begin{array}{ll}
(0,0) & t \notin(a, b) \\
(1,0) & t \in(a, b)
\end{array} .\right.
$$

Note that $\bar{z}$ is not the complex conjugate of $z$ because of the analytic continuation. 


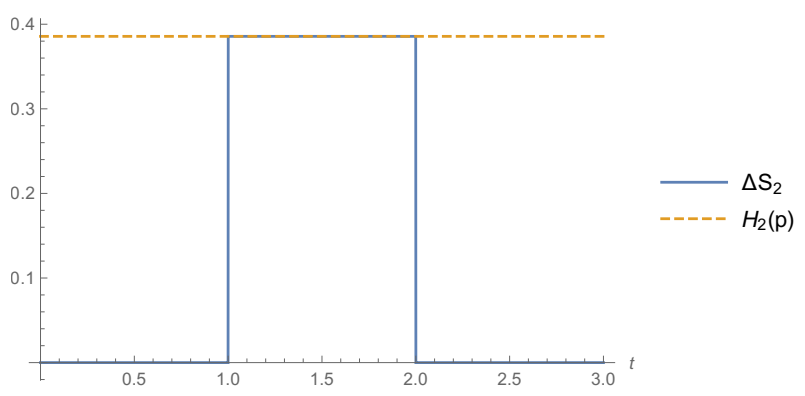

Figure 5. Evolution of the second Rényi entropy for $O_{1}=e^{i \eta \phi}$ and $O_{2}=e^{-i \eta \phi}$ for $p=0.2$.

Similarly, we get (independent of $t$ )

$$
\operatorname{Tr} \rho_{1}^{2}=\operatorname{Tr} \rho_{2}^{2}=\frac{Z_{(2)}}{\left(Z_{(1)}\right)^{2}}
$$

Finally we get

$$
\Delta S_{2}\left(\rho_{A}(t)\right) \equiv S_{2}\left(\rho_{A}(t)\right)-S_{2}\left(\rho_{A}^{0}\right)=\left\{\begin{array}{ll}
0 & t \notin(a, b) \\
-\log \left(p^{2}+(1-p)^{2}\right) & t \in(a, b)
\end{array} .\right.
$$

This is plotted in figure 5. Note that one can understand the behavior in $a<t<b$ by the interpretation of quasi-particle picture of the locally excited states (refer to $[9,10]$ )

$$
e^{i \eta \phi}|\Omega\rangle \simeq|0\rangle, \quad e^{-i \eta \phi}|\Omega\rangle \simeq|1\rangle .
$$

This leads to the interpretation

$$
\rho_{A} \simeq p|0\rangle\langle 0|+(1-p)| 1\rangle\langle 1|,
$$

and this explains the non-trivial entropy in (7.14) for $a<t<b$. In this case the upper bound (7.2) is satisfied because $\rho_{1}$ and $\rho_{2}$ are orthogonal to each other.

On the other hand, when $t>b$ or $t<a$ we find that the lower bound (7.1) is satisfied (though it is not required) because all of the entropies are vanishing and $\rho_{1}$ and $\rho_{2}$ are indistinguishable.

\subsection{The $n$-th Rényi entropy}

Moreover, one can generalize the analysis to the $n$-th Rényi entropy in the $s \rightarrow 0$ limit. In this case, we need to find

$$
\operatorname{Tr}\left[\rho_{i_{1}} \rho_{i_{2}} \cdots \rho_{i_{n}}\right]=\frac{\left\langle O_{i_{1}}^{\dagger}\left(X_{1}, \bar{X}_{1}\right) O_{i_{1}}\left(X_{2}, \bar{X}_{2}\right) \cdots O_{i_{n}}^{\dagger}\left(X_{2 n-1}, \bar{X}_{2 n-1}\right) O_{i_{n}}\left(X_{2 n}, \bar{X}_{2 n}\right)\right\rangle}{\prod_{j=1}^{n} O_{j}^{\dagger}\left(X_{2 j-1}, \bar{X}_{2 j-1}\right) O_{j}\left(X_{2 j}, \bar{X}_{2 j}\right)} \frac{Z_{(n)}}{Z_{(1)}^{n}}
$$

for arbitrary $n \in \mathbb{Z}$ and arbitrary sets of the indices $i_{k}= \pm 1$ for $k \in\{1,2, \ldots, n\}$ (we identify $O_{2} \equiv O_{-1}$ ). 
In the earlier time $t<a$ or the late time $b<t$, the $2 n$-point function appears in (7.17) will factorize in the $s \rightarrow 0$ limit as follows

$$
\begin{gathered}
\left\langle O_{i_{1}}^{\dagger}\left(X_{1}, \bar{X}_{1}\right) O_{i_{1}}\left(X_{2}, \bar{X}_{2}\right) \cdots O_{i_{n}}^{\dagger}\left(X_{2 n-1}, \bar{X}_{2 n-1}\right) O_{i_{n}}\left(X_{2 n}, \bar{X}_{2 n}\right)\right\rangle \\
\sim \prod_{j=1}^{n}\left\langle O_{i_{j}}^{\dagger}\left(X_{2 j-1}, \bar{X}_{2 j-1}\right) O_{i_{j}}\left(X_{2 j}, \bar{X}_{2 j}\right)\right\rangle
\end{gathered}
$$

and thus we find a trivial behavior $\Delta S_{n}\left(\rho_{A}(t)\right)=0$.

On the other hand, for the middle time $a<t<b$, the $2 n$-point function is dominated by a non-trivial factorization

$$
\begin{gathered}
\left\langle O_{i_{1}}^{\dagger}\left(X_{1}, \bar{X}_{1}\right) O_{i_{1}}\left(X_{2}, \bar{X}_{2}\right) \cdots O_{i_{n}}^{\dagger}\left(X_{2 n-1}, \bar{X}_{2 n-1}\right) O_{i_{n}}\left(X_{2 n}, \bar{X}_{2 n}\right)\right\rangle \\
\sim \prod_{j=1}^{n}\left\langle O_{i_{j}}^{\dagger}\left(X_{2 j-1}, \bar{X}_{2 j-1}\right) O_{i_{j}}\left(X_{2 j-2}, \bar{X}_{2 j-2}\right)\right\rangle
\end{gathered}
$$

with the identification $X_{0} \equiv X_{n}$. By using the conformal map

$$
z^{n}=\frac{X-a}{X-b}
$$

we can find (about the chiral part)

$$
\begin{aligned}
\operatorname{Tr}\left[\rho_{i_{1}} \rho_{i_{2}} \cdots \rho_{i_{n}}\right] & \sim \prod_{j=1}^{n}\left(\frac{1}{n^{2 n}} \frac{\left(z_{2 j-1}^{n}-z_{2 j}^{n}\right)^{2}}{z_{2 j-1}^{n-1} z_{2 j}^{n-1}\left(z_{2 j-1}-z_{2 j-2}\right)^{2\left(i_{2 j-1} \cdot i_{2 j-2}\right)}}\right)^{h} \\
& =\left(\varepsilon^{2\left(n-\sum_{j}\left(i_{2 j-1} \cdot i_{2 j-2}\right)\right)} e^{\frac{2 \pi i}{n} \sum_{j}\left(i_{2 j-1} \cdot i_{2 j-2}\right)}\right)^{h}
\end{aligned}
$$

where we have used

$$
z_{2 k+1}=e^{\frac{2 \pi i}{n} k} z_{1}, z_{2 k+2}=e^{\frac{2 \pi i}{n} k} z_{2},
$$

and set $z_{1} \equiv r e^{-\frac{\pi i}{n}+i \varepsilon}$ and $z_{2}=\bar{z}_{1}=r e^{\frac{\pi i}{n}-i \varepsilon}$ with the infinitesimal $\varepsilon$ related to $s$. Thus all the traces will vanish in the $s \rightarrow 0$ limit except the identical cases,

$$
\operatorname{Tr}\left[\rho_{i_{1}} \rho_{i_{2}} \cdots \rho_{i_{n}}\right]=\left\{\begin{array}{ll}
\frac{Z_{(n)}}{Z_{(1)}^{n}} & \text { for } i_{1}=i_{2}=\cdots=i_{n} \\
0 & \text { otherwise }
\end{array} .\right.
$$

Therefore we finally obtain

$$
\Delta S_{n}\left(\rho_{A}(t)\right)=\left\{\begin{array}{ll}
0 & t \notin(a, b) \\
\frac{1}{1-n} \log \left(p^{n}+(1-p)^{n}\right) & t \in(a, b)
\end{array} .\right.
$$

This is exactly what was expected from the quasi-particle picture. The inequalities (7.1) and (7.2) are again saturated as in the second Rényi entropy.

\section{More general mixed states in holographic CFTs}

Finally we use the inequality (1.2) to constrain the evolution of entanglement entropy for mixed states which are obtained by linear combinations of pure state local operator quenches with more general coefficients than the previous one (3.1). We focus on holographic CFTs, to explore any possibilities of gravity duals for general mixed states. 


\subsection{Setup}

We start with the general mixed states of the form,

$$
\rho^{\text {mixed }}=\sum_{i} p\left(\Delta_{i}\right)\left|\Psi_{i}(t)\right\rangle\langle\Psi(t)|
$$

where

$$
p(\Delta)=\frac{e^{-\gamma \Delta^{q}}}{Z} .
$$

Note that $\sum_{i}$ represents a sum of all possible states in a given CFT. We assume $q>1 / 2$ for the convergence of the partition function $Z$. We also focus on the range $q \neq 1$ because $q=1$ is the case which we discussed in the previous sections.

Now to proceed, we first need to normalize $p(\Delta)$ in the following way,

$$
\int_{0}^{\infty} p(\Delta) D(\Delta) d \Delta=1
$$

where $D(\Delta)$ is the density of states. This determines the partition function $Z$. In ideal holographic CFTs, we expect the following behaviors of the density of states:

$$
\begin{array}{rlrl}
D(\Delta) & \approx \mathcal{O}(1), & 0 & <\Delta<\frac{c}{12}, \\
& \approx e^{2 \pi \sqrt{\frac{c}{3}\left(\Delta-\frac{c}{12}\right)}}, & \frac{c}{12}<\Delta<\infty .
\end{array}
$$

Though these requirements are more stronger than the one [44], the details do not affect our arguments in this section. Given this, to compute the Shanon entropy we will need to evaluate the following,

$$
H(p)=-\sum_{i} p_{i} \log p_{i}=-\int_{0}^{\infty} p(\Delta) \log p(\Delta) D(\Delta) d \Delta .
$$

Similarly, to compute the $\sum_{i} p_{i} S\left(\rho_{i}\right)$ we need to evaluate the following,

$$
\sum_{i} p_{i} S\left(\rho_{i}\right)=\int_{0}^{\infty} D(\Delta) p(\Delta) S\left(\rho_{\Delta}\right) d \Delta
$$

The expression for $S\left(\rho_{i}\right)$ is given in (2.7). We will be evaluating the integrals appearing in (8.3), (8.5) and (8.6) by using saddle point approximation in the following section for various choice of $\gamma$.

\subsection{Case 1: $\gamma=\mathcal{O}(1)$}

Let us first assume $\gamma$ remains order one when we take the large $c$ limit $c \rightarrow \infty$. First notice that the saddle point $\Delta=\Delta_{*}$ of the integral of $\Delta$, if exists in the range $\Delta>\frac{c}{12}$, is specified by

$$
\pi \frac{\sqrt{\frac{c}{3}}}{\sqrt{\Delta_{*}-\frac{c}{12}}}-q \gamma \Delta_{*}^{q-1}=0 .
$$


At this saddle point, we can estimate the contribution as follows

$$
p\left(\Delta_{*}\right) D\left(\Delta_{*}\right) \sim \frac{1}{Z} e^{-\gamma \Delta_{*}^{q}+\frac{2 \pi^{2}}{3 q \gamma} c \Delta_{*}^{1-q}} .
$$

Let us start with the case $q>1$. In this case, since $p\left(\Delta_{*}\right) D\left(\Delta_{*}\right) \sim e^{-\mathcal{O}\left(c^{q}\right)} / Z$ at the saddle point $\Delta_{*}>\frac{c}{12}$, we find that the dominant contribution comes from the region $\Delta \in\left[0, \frac{c}{12}\right]$. In this low energy region, due to the exponential damping factor of $p(\Delta)$, the integral is localized around at $\Delta=\mathcal{O}(1)$. At the same time, for the integral (8.5), this clearly means that $H(p)=\mathcal{O}(1)$. Then the sum of entropy (8.6) is also dominated by the contribution around $\Delta=\mathcal{O}(1)$. Since we know that the entanglement entropy $S_{A}$ for the state $\left|\Psi_{i}(t)\right\rangle$ remains the same as that of the ground state in such a case, the same is true for $\sum_{i} p_{i} S\left(\rho_{i}\right)$. Note also that the inequalities (1.2) get very tight because $H(p)=\mathcal{O}(1) \ll \sum_{i} p_{i} S\left(\rho_{i}\right)=\mathcal{O}(c)$. In summary, for $q>1$, we find the rather trivial result:

$$
S\left(\rho_{A}^{\text {mixed }}\right) \simeq \sum_{i} p_{i} S\left(\rho_{i}\right) \simeq \frac{c}{3} \log \left(\frac{b-a}{\epsilon}\right) .
$$

For $1 / 2<q<1$ the dominant contribution comes from the region $\Delta \in\left[\frac{c}{12}, \infty\right]$. The saddle point equation (8.7) shows that

$$
\Delta_{*} \simeq\left(\frac{\pi^{2}}{3 q^{2} \gamma^{2}} c\right)^{\frac{1}{2 q-1}} \gg \mathcal{O}(c) .
$$

Then we get the estimations,

$$
H(p) \approx \frac{2 \pi}{\sqrt{3}} \sqrt{c\left(\frac{c \pi^{2}}{3 \gamma^{2} q^{2}}\right)^{\frac{1}{2 q-1}}}=\mathcal{O}\left(c^{\frac{q}{2 q-1}}\right),
$$

and

$$
\sum_{i} p_{i} S\left(\rho_{i}\right) \approx c^{\frac{q}{2 q-1}}\left(\pi^{2} / 3\right)^{\frac{q}{2 q-1}}(\gamma q)^{-\frac{1}{2 q-1}}=\mathcal{O}\left(c^{\frac{q}{2 q-1}}\right)
$$

where we evaluated the last term in (2.7).

In the above calculations, we have only kept the leading order term in the large $c$ limit. Now we see that expressions in (8.11) and (8.12) are of the same magnitude, hence $S\left(\rho_{P}\right)$ cannot be approximated by $\sum_{i} p_{i} S\left(\rho_{i}\right)$. However, one might speculate $S\left(\rho_{A}^{\text {mixed }}\right)=$ $\mathcal{O}\left(c^{\frac{q}{2 q-1}}\right)$ from the bounds (1.2). Since $\frac{q}{2 q-1}>1$, these contributions contradict with the expectations from the classical gravity description. Also we cannot trust the formula (2.7) which was obtained from the standard large $c$ approximation in such a high energetic setup. In this way, for the range $1 / 2<q<1$, we cannot make any definite prediction for $S\left(\rho_{A}^{\text {mixed }}\right)$ from these arguments.

\subsection{Case 2: $\gamma=\lambda c^{1-q}$}

The previous difficulty in the case $1 / 2<q<1$ motivates us to scale the coefficient $\gamma$ in (8.2) appropriately in the large $c$ limit as follows

$$
\gamma=\lambda c^{1-q},
$$


such that the saddle point is $\Delta_{*}=\mathcal{O}(c)$ and the contributions of various quantities are also $\mathcal{O}(c)$. The constant $\lambda$ in the above equation is taken to be $\mathcal{O}(1)$.

In this setup, we always find a dominant saddle point $\Delta=\Delta_{*}>\frac{c}{12}$ such that $\Delta_{*}$ is $\mathcal{O}(c)$ as the solution to (8.7). When $q \lambda$ is very large or small, we find the behaviors

$$
\begin{array}{ll}
\text { When } q \lambda \ll 1: & \Delta_{*} \simeq\left(\frac{\pi^{2}}{3 q^{2} \lambda^{2}}\right)^{\frac{1}{2 q-1}} c, \\
\text { When } q \lambda \gg 1: \quad \Delta_{*} \simeq \frac{c}{12} .
\end{array}
$$

We can evaluate (8.5) and (8.6) as before and we find that both $H(p)$ and $\sum_{i} p_{i} S\left(\rho_{i}\right)$ are $\mathcal{O}(c)$ in the large $c$ limit. In particular we obtain the behaviors:

$$
\begin{aligned}
& \text { When } q \lambda \ll 1: \quad H(q) \simeq \frac{2 \pi^{2}}{3 \lambda q} \cdot\left(\frac{\pi^{2}}{3 q^{2} \lambda^{2}}\right)^{\frac{1-q}{2 q-1}} \cdot c, \\
& \text { When } q \lambda \gg 1: \quad H(q) \simeq \frac{2 \cdot 12^{q-1} \cdot \pi^{2}}{3 \lambda q} \cdot c
\end{aligned}
$$

and

$$
\begin{aligned}
& \text { When } q \lambda \ll 1: \quad \sum_{i} p_{i} S\left(\rho_{i}\right) \simeq \frac{c}{3} \log \left(\frac{b-a}{\epsilon}\right)+\frac{c}{6} \log \frac{t}{s}+\left(\pi^{2} / 3\right)^{\frac{q}{2 q-1}}(\lambda q)^{-\frac{1}{2 q-1}} \cdot c, \\
& \text { When } q \lambda \gg 1: \quad \sum_{i} p_{i} S\left(\rho_{i}\right) \simeq \frac{c}{3} \log \left(\frac{b-a}{\epsilon}\right)+\frac{c}{6} \log \frac{t}{s}+\frac{c}{6} \log \pi .
\end{aligned}
$$

The inequalities (1.2) tells us that in the present case the entanglement entropy grows logarithmically:

$$
S\left(\rho_{A}^{\text {mixed }}\right) \simeq \frac{c}{3} \log \left(\frac{b-a}{\epsilon}\right)+\frac{c}{6} \log \frac{t}{s}+S_{1}
$$

where $S_{1}$ is a $\mathcal{O}(c)$ constant, though the inequalities are not enough tight to fix $S_{1}$ precisely. Also, the fact that the entanglement entropy is $\mathcal{O}(c)$ suggests a possibility that we can have a classical gravity dual of this mixed state.

\section{Conclusions}

In this paper, we introduced a new class of local quenches called universal local operator quenches. This is a mixed state counterpart of the standard local operator quenches [9, 10], summing over infinitely many of the latter. These local quenches are parameterized by two parameters $\beta$ and $s$, where $\beta$ plays a role of potential for the conformal dimensions of local operators we sum over, and $s$ is a regularization parameter of the local operators. After a conformal mapping, we found that it is described by a path-integral on a torus. Thus the entanglement entropy under a universal local operator quench can be computed as the two point functions of twist operators on a torus. This allows us concrete computations of the time evolution of entanglement entropy. Note that our universal local operator quenches provide rare examples of mixed states for which we can analytically perform calculations using conformal field theoretic methods. 
For explicit calculations of entanglement entropy, we focused on the two different CFTs in two dimensions: holographic CFTs and the massless Dirac fermion CFT. In holographic CFTs, our results of entanglement entropy under universal local operator quenches coincide with known results for the standard (pure state) local operator quenches, by relating $\beta$ in the former to the conformal dimension $\Delta$ of the local operator in the latter via (6.9). Both of them grow logarithmically $\sim \frac{c}{6} \log t$ at late time under the time evolutions. We can conclude that this coincidence between the mixed state results and the pure state results is due to the chaotic nature of holographic CFTs. In other words, this is a consequence of ETH (eigenstate thermalization hypothesis) [47, 48] for two dimensional holographic CFTs.

In the massless Dirac Fermion CFT, on the other hand, we found new behaviors from the time evolutions of entanglement entropy under universal local operator quenches. At late time, the growth of entanglement entropy approaches to a constant given by $\frac{1}{2} S_{t h}(\beta)$, where $S_{t h}$ is the thermal entropy when we consider the thermal partition function $Z(\beta)=$ $\sum_{i} e^{-\beta\left(\Delta_{i}-c / 12\right)}$. This makes an intriguing contrast with the results for a local quench by a single primary operator, where the growth of entanglement entropy approaches to a constant given by the quantum dimension [14].

We also found a common property for both the holographic and Dirac fermion CFT when $\beta$ is very small. In this case there is an intermediate time region $s \ll t \ll s / \beta$, where the entanglement entropy grows logarithmically $\sim \frac{c}{3} \log t$ with the doubled coefficient.

Moreover, in the latter parts of this paper, we discussed the implication of inequalities (1.2). In holographic CFTs, we found that one of inequalities is saturated, which is understood because we cannot distinguish various pure states due to the chaotic nature. In the Dirac fermion CFT, we obtain an upper bound from the inequality. This clearly proves that the entanglement entropy under pure state local operator quenches for any operator does not grow logarithmically as opposed to the entanglement entropy in holographic CFTs. We also discussed analogous inequalities for Rényi entropy and analyzed a simple example of a free scalar CFT, where the saturations of inequalities occur.

Finally we explored what we can predict for the entanglement entropy in more general mixed states, by employing the inequalities (1.2), when the probability distributions take the form $p_{i} \propto e^{-\gamma \Delta^{q}}$. Even though in general the analysis goes beyond our current knowledge, we manage to find a controllable example by fine-tuning $\gamma$ such that the resulting entanglement entropy is $O(c)$ and the bounds (1.2) provide meaningful predictions.

This paper initiates studies of entanglement entropy in general mixed states other than the standard finite temperature state. We expect such a research direction is also important for deeper understandings of AdS/CFT in order to work out what quantum states in holographic CFTs can have classical gravity duals. We can also think of various generalizations of our analysis in this paper, such as universal local operator quenches in higher dimensional CFTs and multiple local operator quenches. We would like to leave these for future problems. 


\section{Acknowledgments}

We are grateful to Pawel Caputa, Yoshifumi Nakata, Yuya Kusuki and Tomonori Ugajin for useful comments. TT is supported by the Simons Foundation through the "It from Qubit" collaboration and by World Premier International Research Center Initiative (WPI Initiative) from the Japan Ministry of Education, Culture, Sports, Science and Technology (MEXT). AB and TT are supported by JSPS Grant-in-Aid for JSPS fellows 17F17023. TT is supported by JSPS Grant-in-Aid for Scientific Research (A) No.16H02182 and by JSPS Grant-in-Aid for Challenging Research (Exploratory) 18K18766. KU is supported by Grant-in-Aid for JSPS Fellows No.18J22888.

Open Access. This article is distributed under the terms of the Creative Commons Attribution License (CC-BY 4.0), which permits any use, distribution and reproduction in any medium, provided the original author(s) and source are credited.

\section{References}

[1] P. Calabrese and J. Cardy, Entanglement entropy and conformal field theory, J. Phys. A 42 (2009) 504005 [arXiv:0905.4013] [INSPIRE].

[2] H. Casini and M. Huerta, Entanglement entropy in free quantum field theory, J. Phys. A 42 (2009) 504007 [arXiv: 0905.2562] [INSPIRE].

[3] T. Nishioka, Entanglement entropy: holography and renormalization group, Rev. Mod. Phys. 90 (2018) 035007 [arXiv: 1801.10352] [INSPIRE].

[4] S. Ryu and T. Takayanagi, Holographic derivation of entanglement entropy from AdS/CFT, Phys. Rev. Lett. 96 (2006) 181602 [hep-th/0603001] [INSPIRE].

[5] S. Ryu and T. Takayanagi, Aspects of Holographic Entanglement Entropy, JHEP 08 (2006) 045 [hep-th/0605073] [INSPIRE].

[6] V.E. Hubeny, M. Rangamani and T. Takayanagi, A Covariant holographic entanglement entropy proposal, JHEP 07 (2007) 062 [arXiv:0705.0016] [INSPIRE].

[7] M.A. Niesen and I.L. Chuang, Quantum Computations and Quantum Information, Cambridge University Press, (2000).

[8] A. Almheiri, X. Dong and B. Swingle, Linearity of Holographic Entanglement Entropy, JHEP 02 (2017) 074 [arXiv: 1606.04537] [INSPIRE].

[9] M. Nozaki, T. Numasawa and T. Takayanagi, Quantum Entanglement of Local Operators in Conformal Field Theories, Phys. Rev. Lett. 112 (2014) 111602 [arXiv:1401.0539] [InSPIRE].

[10] M. Nozaki, Notes on Quantum Entanglement of Local Operators, JHEP 10 (2014) 147 [arXiv: 1405.5875] [INSPIRE].

[11] P. Calabrese and J. Cardy, Entanglement and correlation functions following a local quench: a conformal field theory approach, J. Stat. Mech. 0710 (2007) P10004 [arXiv:0708.3750] [INSPIRE].

[12] T. Shimaji, T. Takayanagi and Z. Wei, Holographic Quantum Circuits from Splitting/Joining Local Quenches, JHEP 03 (2019) 165 [arXiv: 1812.01176] [INSPIRE].

[13] T. Ugajin, Two dimensional quantum quenches and holography, arXiv:1311.2562 [INSPIRE]. 
[14] S. He, T. Numasawa, T. Takayanagi and K. Watanabe, Quantum dimension as entanglement entropy in two dimensional conformal field theories, Phys. Rev. D 90 (2014) 041701 [arXiv: 1403.0702] [INSPIRE].

[15] M. Nozaki, T. Numasawa and T. Takayanagi, Holographic Local Quenches and Entanglement Density, JHEP 05 (2013) 080 [arXiv:1302.5703] [INSPIRE].

[16] C.T. Asplund, A. Bernamonti, F. Galli and T. Hartman, Holographic Entanglement Entropy from 2d CFT: Heavy States and Local Quenches, JHEP 02 (2015) 171 [arXiv:1410.1392] [INSPIRE].

[17] C. Holzhey, F. Larsen and F. Wilczek, Geometric and renormalized entropy in conformal field theory, Nucl. Phys. B 424 (1994) 443 [hep-th/9403108] [InSPIRE].

[18] P. Caputa, M. Nozaki and T. Takayanagi, Entanglement of local operators in large- $N$ conformal field theories, PTEP 2014 (2014) 093B06 [arXiv: 1405.5946] [INSPIRE].

[19] P. Caputa, J. Simón, A. Štikonas and T. Takayanagi, Quantum Entanglement of Localized Excited States at Finite Temperature, JHEP 01 (2015) 102 [arXiv:1410.2287] [INSPIRE].

[20] J. de Boer, A. Castro, E. Hijano, J.I. Jottar and P. Kraus, Higher spin entanglement and $\mathcal{W}_{\mathrm{N}}$ conformal blocks, JHEP 07 (2015) 168 [arXiv:1412.7520] [INSPIRE].

[21] W.-Z. Guo and S. He, Rényi entropy of locally excited states with thermal and boundary effect in 2D CFTs, JHEP 04 (2015) 099 [arXiv: 1501.00757] [INSPIRE].

[22] B. Chen, W.-Z. Guo, S. He and J.-q. Wu, Entanglement Entropy for Descendent Local Operators in 2D CFTs, JHEP 10 (2015) 173 [arXiv:1507.01157] [INSPIRE].

[23] M. Nozaki, T. Numasawa and S. Matsuura, Quantum Entanglement of Fermionic Local Operators, JHEP 02 (2016) 150 [arXiv:1507.04352] [INSPIRE].

[24] P. Caputa and A. Veliz-Osorio, Entanglement constant for conformal families, Phys. Rev. D 92 (2015) 065010 [arXiv: 1507.00582] [INSPIRE].

[25] P. Caputa, J. Simón, A. Štikonas, T. Takayanagi and K. Watanabe, Scrambling time from local perturbations of the eternal BTZ black hole, JHEP 08 (2015) 011 [arXiv:1503.08161] [INSPIRE].

[26] M. Rangamani, M. Rozali and A. Vincart-Emard, Dynamics of Holographic Entanglement Entropy Following a Local Quench, JHEP 04 (2016) 069 [arXiv: 1512.03478] [INSPIRE].

[27] A. Sivaramakrishnan, Localized Excitations from Localized Unitary Operators, Annals Phys. 381 (2017) 41 [arXiv:1604.00965] [INSPIRE].

[28] P. Caputa and M.M. Rams, Quantum dimensions from local operator excitations in the Ising model, J. Phys. A 50 (2017) 055002 [arXiv: 1609.02428] [InSPIRE].

[29] T. Numasawa, Scattering effect on entanglement propagation in RCFTs, JHEP 12 (2016) 061 [arXiv: 1610.06181] [INSPIRE].

[30] M. Nozaki and N. Watamura, Quantum Entanglement of Locally Excited States in Maxwell Theory, JHEP 12 (2016) 069 [arXiv: 1606.07076] [INSPIRE].

[31] J.R. David, S. Khetrapal and S.P. Kumar, Universal corrections to entanglement entropy of local quantum quenches, JHEP 08 (2016) 127 [arXiv: 1605.05987] [INSPIRE].

[32] P. Caputa, Y. Kusuki, T. Takayanagi and K. Watanabe, Evolution of Entanglement Entropy in Orbifold CFTs, J. Phys. A 50 (2017) 244001 [arXiv:1701.03110] [INSPIRE]. 
[33] M. Nozaki and N. Watamura, Correspondence between entanglement growth and probability distribution of quasiparticles, Phys. Rev. D 96 (2017) 025019 [arXiv:1703.06589] [INSPIRE].

[34] A. Jahn and T. Takayanagi, Holographic entanglement entropy of local quenches in AdS4/CFT3: a finite-element approach, J. Phys. A 51 (2018) 015401 [arXiv:1705.04705] [INSPIRE].

[35] S. He, Conformal bootstrap to Rényi entropy in 2D Liouville and super-Liouville CFTs, Phys. Rev. D 99 (2019) 026005 [arXiv:1711.00624] [INSPIRE].

[36] Y. Kusuki and T. Takayanagi, Rényi entropy for local quenches in 2D CFT from numerical conformal blocks, JHEP 01 (2018) 115 [arXiv:1711.09913] [INSPIRE].

[37] Y. Kusuki, Light Cone Bootstrap in General 2D CFTs and Entanglement from Light Cone Singularity, JHEP 01 (2019) 025 [arXiv:1810.01335] [INSPIRE].

[38] L. Apolo, S. He, W. Song, J. Xu and J. Zheng, Entanglement and chaos in warped conformal field theories, JHEP 04 (2019) 009 [arXiv: 1812.10456] [INSPIRE].

[39] Y. Kusuki and M. Miyaji, Entanglement Entropy, OTOC and Bootstrap in 2D CFTs from Regge and Light Cone Limits of Multi-point Conformal Block, JHEP 08 (2019) 063 [arXiv: 1905.02191] [INSPIRE].

[40] P. Caputa, T. Numasawa, T. Shimaji, T. Takayanagi and Z. Wei, Double Local Quenches in 2D CFTs and Gravitational Force, JHEP 09 (2019) 018 [arXiv:1905.08265] [INSPIRE].

[41] S. He and H. Shu, Correlation functions, entanglement and chaos in the $T \bar{T} / J \bar{T}$-deformed CFTs, arXiv: 1907.12603 [INSPIRE].

[42] Y. Kusuki and M. Miyaji, Entanglement Entropy after Double-Excitation as Interaction Measure, arXiv:1908.03351 [INSPIRE].

[43] T. Azeyanagi, T. Nishioka and T. Takayanagi, Near Extremal Black Hole Entropy as Entanglement Entropy via $A d S_{2} / C F T_{1}$, Phys. Rev. D 77 (2008) 064005 [arXiv:0710. 2956] [INSPIRE].

[44] T. Hartman, C.A. Keller and B. Stoica, Universal Spectrum of 2d Conformal Field Theory in the Large c Limit, JHEP 09 (2014) 118 [arXiv:1405.5137] [INSPIRE].

[45] N. Bao and H. Ooguri, Distinguishability of black hole microstates, Phys. Rev. D 96 (2017) 066017 [arXiv: 1705. 07943] [INSPIRE].

[46] G.M. Bosyk, S. Zozor, F. Holik, M. Portesi and P.W. Lamberti, A family of generalized quantum entropies: definition and properties, Quant. Inf. Proc. 15 (2016) 3393.

[47] M. Srednicki, The Approach to Thermal Equilibrium in Quantized Chaotic Systems, J. Phys. A 32 (1999) 1163 [cond-mat/9809360].

[48] A.L. Fitzpatrick, J. Kaplan and M.T. Walters, Universality of Long-Distance AdS Physics from the CFT Bootstrap, JHEP 08 (2014) 145 [arXiv: 1403.6829] [INSPIRE]. 